\title{
Increased susceptibility to ischemia-induced ventricular tachyarrhythmias in depressed rats: Involvement of reduction of connexin 43
}

\author{
WEI WU ${ }^{1}$, YAN LI $^{1}$, ZHIBING LU ${ }^{2}$ and XIAORONG HU ${ }^{2}$ \\ Departments of ${ }^{1}$ Clinical Laboratory, and ${ }^{2}$ Cardiology, Renmin Hospital of Wuhan University, Wuhan, P.R. China
}

Received September 21, 2011; Accepted November 11, 2011

DOI: $10.3892 /$ etm.2011.396

\begin{abstract}
Connexin $43(\mathrm{Cx} 43)$ has been reported to contribute to the occurrence of ventricular arrhythmias during myocardial ischemia (MI). In this study, we investigated the expression of Cx43 and the incidences of ventricular tachyarrhythmias [i.e., ventricular tachycardia (VT) and ventricular fibrillation (VF)] during acute MI in chronic mild stress (CMS) in rats. Male Sprague-Dawley (SD) control and CMS rats were assigned into a sham operation (SO) group and a MI group. Ventricular tachyarrhythmias were assessed and Cx43 protein expression was measured by Western blotting. During 30-min ischemia, the incidences of VT $(7 / 12,58.3 \%)$ and VF $(5 / 12,41.7 \%)$ in the CMS-MI group were significantly decreased compared with those in the control-MI group (12/12, 100.0\% and 11/12,91.7\%; $\mathrm{P}<0.05)$. The amount of total $\mathrm{Cx} 43$ of the CMS-SO group was significantly decreased to approximately $50 \%$ compared with that of the control-SO group $(\mathrm{P}<0.05)$. The 30-min ischemia did not result in a significant change in the amount of total Cx43 (total Cx43 is defined as the non-phosphorylated Cx43 and phosphorylated $\mathrm{Cx} 43$ ) compared to that of the SO group in CMS rats $(\mathrm{P}>0.05)$. The amount of non-phosphorylated $\mathrm{Cx} 43$ in the CMS-MI group was markedly increased compared to that of the CMS-SO group $(\mathrm{P}<0.05)$, suggesting that the relative amount of phosphorylated $\mathrm{Cx} 43$ was significantly decreased in CMS rats. The gap junctional permeability in the CMS-MI group $(50.4 \pm 4.9 \%)$ was significantly decreased compared with the normal non-ischemic value in the CMS-SO group $(100 \%)$. The present study suggested that the incidence of ischemia-induced ventricular tachyarrhythmias was markedly increased in depressed rats, which may be associated with the reduction of $\mathrm{Cx} 43$ protein expression in the ventricle of depressed rats.
\end{abstract}

Correspondence to: Dr Xiaorong Hu, Department of Cardiology, Renmin Hospital of Wuhan University, 238 Jiefang Road, Wuchang, Wuhan 430060, P.R. China

E-mail: huxrwurm@163.com

Key words: connexin 43, ventricular arrhythmia, myocardial ischemia, depression

\section{Introduction}

Intercellular electrical coupling occurs through protein conduits and gap junctions that are formed by connexins. In the heart, the most important connexins are connexins $40,43(\mathrm{Cx} 43)$ and 45 , while $\mathrm{Cx} 43$ is the main electrical coupling protein in the ventricles (1). Cx43 has been reported to contribute to the occurrence of ventricular arrhythmias during myocardial ischemia (MI) (2,3).

Grippo et al (4) demonstrated that chronic mild stress (CMS) rats exhibit an increased vulnerability to ventricular arrhythmias induced by electrical stimulation. However, the mechanism behind this remains unclear. Recent studies have revealed that alterations in the amount of Cx43 and Cx43 phosphoralation status are important in the genesis of ventricular arrhythmias during acute MI $(5,6)$. In the present study, we investigated the expression of $\mathrm{Cx} 43$ and the incidences of ventricular tachyarrhythmias [i.e., ventricular tachycardia (VT) and ventricular fibrillation (VF)] during acute MI in CMS rats.

\section{Materials and methods}

Experimental animals. The experiment protocol conformed to the Guideline for the Care and Use of Laboratory Animals published by the US National Institutes of Health (NIH Publication, revised 1996) and was approved by the Institutional Animal Care and Use Committee. Male Sprague-Dawley (SD) rats were randomly assigned into the following groups: MI group (control-MI, $n=12$ ), ligation of the left anterior descending (LAD) coronary artery; sham operation (SO) group (control-SO, n=12), without coronary ligation; CMS + SO group (CMS-SO, n=12), without coronary ligation; CMS + MI group (CMS-MI, n=12), ligation of the LAD coronary artery. The CMS model was designed according to a previously described method (4).

VT was assessed and defined as $\geq 10$ ventricular ectopic beats with a cycle length $<100 \mathrm{~ms}$; VF was defined as unidentifiable and low voltage QRS complexes; VF may be sustained or may revert spontaneously to normal sinus rhythm (5).

Immunoblot analysis. Regions of pulverized frozen ischemia from left ventricle samples were analyzed by quantitative immunoblotting using a goat polyclonal anti-Cx43 antibody 
(sc-56698; Santa Cruz Biotechnology, CA, USA) for total Cx43 protein and a non-phosphorylated mouse monoclonal antiCx43 antibody (13-8300; Zymed/Invitrogen, Carlsbad, CA, USA) for non-phosphorylated $\mathrm{Cx} 43$ content. The expressions of non-phosphorylated and total $\mathrm{Cx} 43$ protein were normalized to glyceraldehyde-3-phosphate dehydrogenase (GAPDH) expression. The relative amounts of non-phosphorylated and total $\mathrm{Cx} 43$ protein were expressed as a percentage of the SO group.

Immunofluorescence. For immunofluorescence of $\mathrm{Cx} 43$, a polyclonal rabbit anti-Cx43 antibody (Santa Cruz Biotechnology) was used. Frozen hearts were sectioned into $6 \mu$ m-thick slices; following permeabilization ( $0.25 \%$ Triton X-100), quenching and blocking (10\% goat serum), samples were incubated with the antibody [1:100 diluted in phosphate-buffered saline (PBS)] overnight at room temperature. Primary antibody-bound $\mathrm{Cx} 43$ was visualized by fluorescein isothiocyanate (FITC)-conjugated anti-rabbit IgG and examined using a microscope (Leica, Germany).

Measurement of gap junctional permeability. Gap junctional permeability values, which were obtained from 5 samples in each group, were assessed using double dye-loading (6). The ratio of Lucifer yellow and rhodamine-dextran stained areas was calculated using a computer-assisted image analysis system (Image-Pro Plus 3.0). Gap junctional permeability within the ischemic area was expressed as the percentage of permeability measured within the non-ischemic area of the SO group.

Statistical analysis. Statistical analysis was performed using SPSS 13.0 software (SPSS Inc., Chicago, IL, USA). All values were expressed as the means \pm SD or the percentage of incidence. The Student's t-test was used for comparisons between the two groups. Fisher's exact test or the Chi-square test were used for comparisons of the incidences of ventricular tachyarrhythmias (i.e., VT and VF). A P-value of $<0.05$ was considered to indicate a statistically significant difference.

\section{Results}

Incidences of VT and VF. The incidences of VT (7/12, 58.3\%) and $\operatorname{VF}(5 / 12,41.7 \%)$ in the CMS-MI group were significantly decreased compared to those in the control-MI group (12/12, $100.0 \%$ and $11 / 12,91.7 \%$; both $\mathrm{P}<0.05)$.

Changes in CX43 protein expression. As shown in Fig. 1A, the amount of total $\mathrm{Cx} 43$ in the CMS-SO group was significantly decreased to $\sim 50 \%$ compared to that in the control-SO group $(\mathrm{P}<0.05)$. Immunofluorescence also revealed that the amount of total Cx43 in the CMS-SO group was significantly decreased compared to that in the control-SO group $(\mathrm{P}<0.05$; Fig. 2).

As shown in Fig. 1B, the 30-min ischemia did not result in a significant change in the amount of total $\mathrm{Cx} 43$ (total $\mathrm{Cx} 43=$ non-phosphorylated $\mathrm{Cx} 43$ + phosphorylated $\mathrm{Cx} 43$ ) in the CMS rats $(\mathrm{P}>0.05)$. The amount of non-phosphorylated $\mathrm{Cx} 43$ in the CMS-MI group was markedly increased compared to that in the CMS-SO group $(\mathrm{P}<0.05)$, suggesting that the relative
A

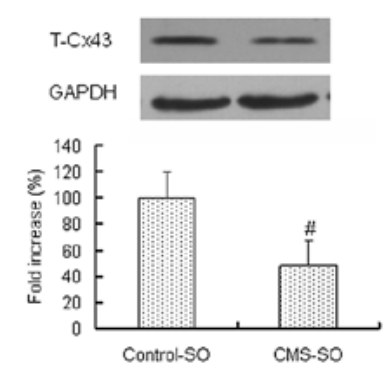

$\mathbf{B}$
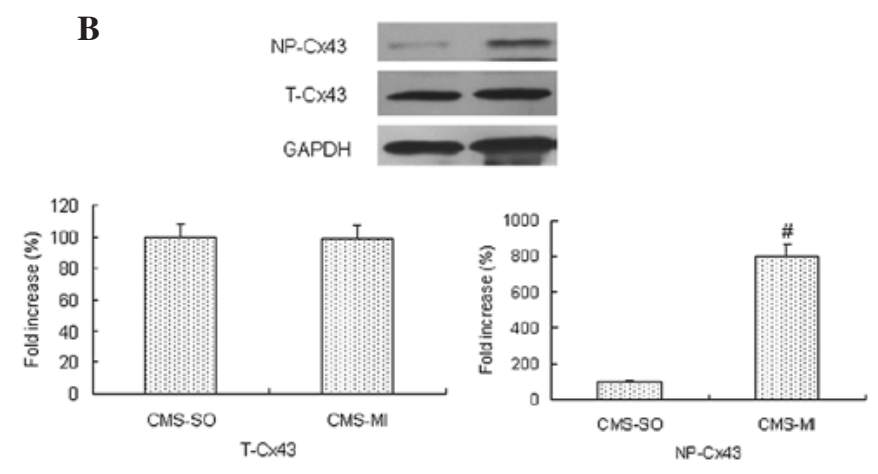

Figure 1. Representative immunoblots of the Cx43 protein. Quantitative densitometric analysis of the amount of NP-Cx43 and T-Cx43 normalized to GAPDH (NP-Cx43/GAPDH; T-Cx43/GAPDH). The relative amounts of NP-Cx43 and T-Cx43 were expressed as a percentage of the control-SO or CMS-SO groups. (A) Comparison of the amount of total $\mathrm{Cx} 43$ between the control-SO and CMS-SO groups. ${ }^{\text {" }} \mathrm{P}<0.05$ vs. control-SO group. (B) Comparison of the amount of total Cx43 and NP-Cx43 between the control-SO and CMS-MI groups in CMS rats. ${ }^{\text {"P }}<0.05$ vs. CMI-SO group. T-Cx43 = NP-Cx43 + P-Cx43. NP-Cx43, non-phosphorylated Cx43; P-Cx43, phosphorylated $\mathrm{Cx} 43$; T-Cx43, total Cx43; GAPDH, glyceraldehyde-3phosphate dehydrogenase; SO, sham-operated; MI, myocardial ischemia; CMS, chronic mild stress; Cx43, connexin 43.

amount of phosphorylated Cx43 was significantly decreased in the CMS rats.

Changes in gap junctional permeability. As shown in Fig. 3, following a 30-min LAD ligation, the gap junctional permeability in the CMS-MI group $(50.4 \pm 4.9 \%)$ was significantly decreased compared with the normal non-ischemic value in the CMS-SO group (100\%).

\section{Discussion}

The prevalence of depression in patients with coronary artery disease (such as myocardial infarction and heart failure) is approximately 5 times that of the general population (7). In the present study, we found that CMS rats were susceptible to ischemia-induced ventricular tachyarrhythmias. This indicates that depression may be a cause of susceptibility to the occurrence of ventricular arrhythmias.

Previous studies have demonstrated that reduced expression of $\mathrm{Cx} 43$ results in a significant reduction in conduction velocity during acute MI and accelerates the onset and increases the incidence of ischemia-induced ventricular arrhythmias $(2,3,8-10)$. These results suggest that the changes in Cx43 expression may play a significant role in the genesis of ventricular arrhythmias during acute MI. Other studies have also shown that $\mathrm{Cx} 43$ underwent marked dephosphorylation during the process of electrical uncoupling induced by 

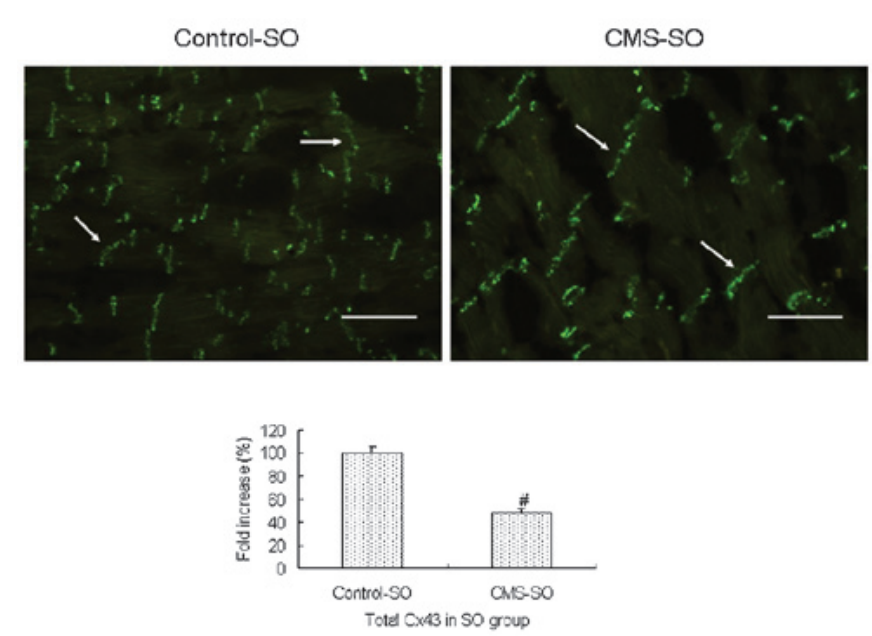

Figure 2. Representative immunofluorescence images of the $\mathrm{Cx} 43$ protein. SO, sham-operated; MI, myocardial ischemia; CMS, chronic mild stress. ${ }^{\#} \mathrm{P}<0.05$ vs. control-SO group. Bars, $100 \mu \mathrm{m}$. Cx43, connexin 43.

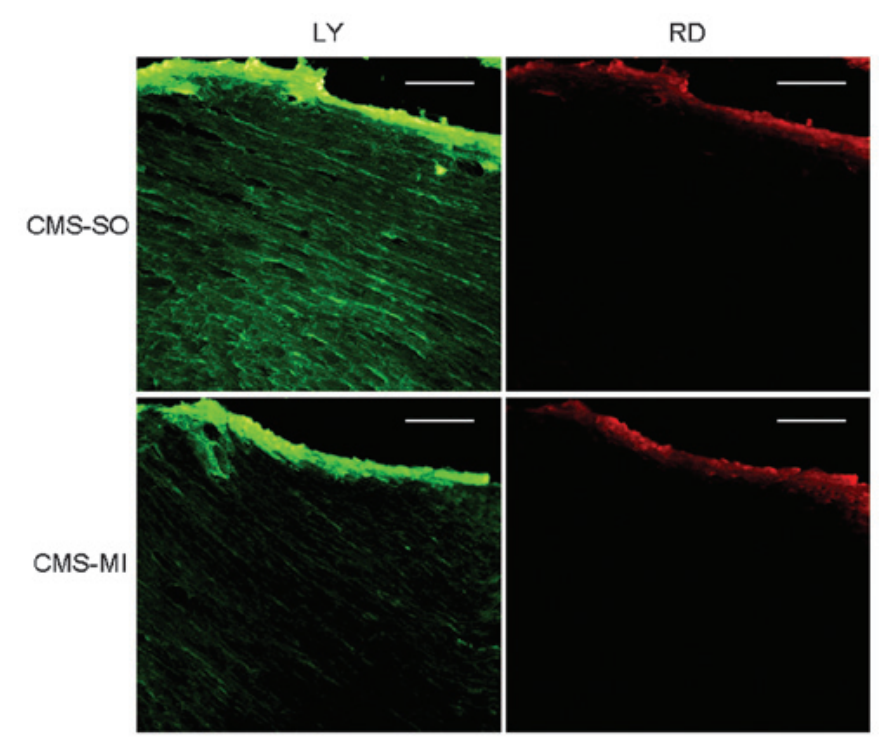

Figure 3. Representative example of gap junctional permeability. LY, Lucifer yellow; RD, rhodamine-dextran; SO, sham-operated; MI, myocardial ischemia; CMS, chronic mild stress. Bars, $100 \mu \mathrm{m}$.

ischemia, which promoted the genesis of ischemia-induced ventricular arrhythmias $(6,11)$. In the present study, the amount of total Cx43 in the CMS-SO group was significantly decreased to approximately $50 \%$ compared to that in the control-SO group. In addition, we found that ischemia did result in $\mathrm{Cx} 43$ dephosphorylation and that the gap junctional permeability, which could directly mediate the electrical coupling and conduction (6) in the MI group, was significantly decreased in the SO group in CMS rats, indicating that the relative amount of phosphorylated $\mathrm{Cx} 43$ in CMS rats was significantly lower than that in adult rats during MI $(5,10)$. These results suggest that the increased susceptibility to ischemia-induced ventricular tachyarrhythmias may be associated with a reduction in $\mathrm{Cx} 43$ protein expression in depressed rats.
This study had several limitations, however. We first observed that the incidence of ischemia-induced ventricular tachyarrhythmias was markedly increased and that the Cx43 protein expression in the ventricle was significantly decreased in depressed rats; however, we did not have direct evidence demonstrating the relationship between the alteration in $\mathrm{Cx} 43$ expression and the occurrence of VT/VF, which was also a limitation of a previous study (10).

In conclusion, the present study suggests that the incidence of ischemia-induced ventricular tachyarrhythmias is markedly increased in depressed rats, which may be associated with the reduction in $\mathrm{Cx} 43$ protein expression in the ventricle of depressed rats.

\section{Acknowledgements}

This study was supported by grant no. 81070143 (to Z.L.) from the National Natural Science Foundation of China, grant no. 4101024 from the Fundamental Research Funds for the Central Universities (to Z.L.) and the Specialized Research Fund for the Doctoral Program of Higher Education of China (grant no. 20100141120072, to Z.L.).

\section{References}

1. Boengler K, Schulz R and Heusch G: Connexin 43 signalling and cardioprotection. Heart 92: 1724-1727, 2006.

2. Smith WT IV, Fleet WF, Johnson TA, Engle CL and Cascio WE: The Ib phase of ventricular arrhythmias in ischemic in situ porcine heart is related to changes in cell-to-cell electrical coupling. Experimental Cardiology Group, University of North Carolina. Circulation 92: 3051-3060, 1995.

3. Saffitz JE, Schuessler RB and Yamada KA: Mechanisms of remodeling of gap junction distributions and the development of anatomic substrates of arrhythmias. Cardiovasc Res 42: 309-317, 1999.

4. Grippo AJ, Santos CM, Johnson RF, Beltz TG, Martins JB, Felder RB and Johnson AK: Increased susceptibility to ventricular arrhythmias in a rodent model of experimental depression. Am J Physiol Heart Circ Physiol 286: H619-H626, 2004.

5. Wu W and $\mathrm{Lu} \mathrm{Z}$ : Loss of anti-arrhythmic effect of vagal nerve stimulation on ischemia-induced ventricular tachyarrhythmia in aged rats. Tohoku J Exp Med 223: 27-33, 2011.

6. Papp R, Gonczi M, Kovacs M, Seprenyi G and Vegh A: Gap junctional uncoupling plays a trigger role in the antiarrhythmic effect of ischaemic preconditioning. Cardiovasc Res 74: 396-405, 2007.

7. Schleifer SJ, Macari-Hinson MM, Coyle DA, Slater WR, Kahn M, Gorlin R and Zucker HD: The nature and course of depression following myocardial infarction. Arch Intern Med 149: 1785-1789, 1989.

8. Lerner DL, Yamada KA, Schuessler RB and Saffitz JE: Accelerated onset and increased incidence of ventricular arrhythmias induced by ischemia in $\mathrm{Cx} 43$-deficient mice. Circulation 101: 547-552, 2000.

9. Gutstein DE, Morley GE, Tamaddon H, Vaidya D, Schneider MD, Chen J, Chien KR, Stuhlmann H and Fishman GI: Conduction slowing and sudden arrhythmic death in mice with cardiac-restricted inactivation of connexin43. Circ Res 88: 333-339, 2001.

10. Ando M, Katare RG, Kakinuma Y, Zhang D, Yamasaki F, Muramoto $\mathrm{K}$ and Sato T: Efferent vagal nerve stimulation protects heart against ischemia-induced arrhythmias by preserving connexin43 protein. Circulation 112: 164-170, 2005.

11. Jiang H, Hu X, Lu Z, Wen H, Zhao D, Tang Q and Yang B: Effects of sympathetic nerve stimulation on ischemia-induced ventricular arrhythmias by modulating connexin 43 in rats. Arch Med Res 39: 647-654, 2008. 